\title{
A WHITE DWARF COMPANION TO THE B5 V STAR HR 2875 (y PUPPIS) ${ }^{1}$

\author{
Stéphane Vennes, ${ }^{2,3}$ Thomas W. Berghöfer, ${ }^{2}$ and Damian J. Christian ${ }^{4}$ \\ Received 1997 August 14; accepted 1997 October 14; published 1997 November 11
}

\begin{abstract}
An extreme-ultraviolet source in the EUVE and ROSAT Wide Field Camera catalogs, also identified with a very soft ROSAT PSPC X-ray source, is coincident with the B5 V star HR 2875 (y Pup). We present an analysis of PSPC and EUVE spectra revealing the presence of a hot white dwarf companion to HR 2875 , which then constitute the first confirmed B star plus white dwarf system. The B star shows variable Si II, He I, and H I line profiles in high-dispersion spectra, possibly related to its binary nature, but our measurements limit the radial velocity semiamplitude of the B star to $\leq 2 \mathrm{~km} \mathrm{~s}^{-1}$. We speculate that the white dwarf is most likely massive, and we propose some observational tests of the model.
\end{abstract}

Subject headings: binaries: general — stars: abundances — stars: individual (HR 2875) — ultraviolet: stars — white dwarfs

\section{INTRODUCTION}

The star HR 2875 (y Pup, HD 59635, CD -38³400) is a southern hemisphere luminous blue object recently detected at extreme-ultraviolet (EUV) wavelengths (EUVE J0729-388, 2RE J0729-384) ${ }^{5}$ and soft X-ray energy (IRXS J072905.9-384839). ${ }^{6}$ Hiltner, Garrison, \& Schild (1969) classify HR 2875 as a B5 Vp star, which translates into a distance modulus of $m-M=6.5$. This classification is in agreement with the recent Hipparcos parallax measurement, $\pi=$ 0"0056 \pm 0".0005 ( $d=164-196$ pc). Hiltner et al. (1969) noted abnormally strong silicon lines in optical spectra.

Motch et al. (1997) report ROSAT PSPC observations that suggest but do not demonstrate the presence of a white dwarf companion to the B5 V star HR 2875. Berghöfer et al. (1997) also recognized that a few other B stars, besides HR 2875, associated with ultrasoft X-ray sources may, in fact, hide hot white dwarf companions (e.g., $\lambda$ Cen and $\lambda$ Sco). In the case of HR 2875, the progenitor of the white dwarf would have been a main-sequence star earlier than B5 $\left(M \geq 6 M_{\odot}\right)$; some initial-mass-final-mass relations $\left(M_{f} / M_{i}\right)$ applicable to such objects (Vassiliadis \& Wood 1993; Wagenhuber \& Weiss 1994; Blöcker 1995) would predict the presence of a high-mass white dwarf star $\left(M \geq 0.9 M_{\odot}\right)$. A study of the HR 2875 system would, in fact, help constrain theoretical $M_{f} / M_{i}$ relations and would provide useful insights to the evolution of early mainsequence stars.

Using EUV and soft X-ray observations of HR 2875, we propose to demonstrate the presence of a hot white dwarf companion to the B star, and, adopting a new estimate of the distance toward HR 2875, we constrain the white dwarf parameters. In $\S 2$ we present new optical, ultraviolet, and soft X-ray observations of HR 2875 that show the likely presence of a white dwarf. In $\S 3$ we show that the properties of the

\footnotetext{
${ }^{1}$ Based on observations obtained at Lick Observatory, operated by the University of California, Mount Stromlo Observatory, operated by the Australian National University, and with ROSAT and NASA's Extreme Ultraviolet Explorer.

${ }^{2}$ Space Sciences Laboratory, University of California, Berkeley, Berkeley, CA 94720-7450; vennes@ssl.berkeley.edu.

${ }^{3}$ Astrophysical Theory Centre, Australian National University, ACT 0200, Canberra, Australia.

${ }^{4}$ Center for EUV Astrophysics, University of California, Berkeley, 2150 Kittredge Street, Berkeley, CA 94720.

${ }^{5}$ Pye et al. (1995); Bowyer et al. (1996).

${ }^{6}$ Voges et al. (1997).
}

white dwarf resemble in every aspect the properties of the EUVselected population of white dwarf stars. We summarize in $\S$ 4 , discuss the implications for stellar evolution, propose new observations to measure the surface gravity, and hence the mass, of the white dwarf, and determine the binary properties.

\section{OBSERVATIONS}

\subsection{X-Ray}

Berghöfer, Schmitt, \& Cassinelli (1996) and Motch et al. (1997) report ROSAT PSPC observations of an ultrasoft X-ray source associated with the B5 V star HR 2875 between 1990 October 17 and 20 (separation = 5.5, well within the PSPC error circle). Figure 1 shows the analysis of the PSPC energy spectrum with blackbody models revealing a very soft source $(T[\mathrm{eV}]=15.6 \pm 3.7)$ also characterized by a low neutral hydrogen column density in the line of sight $\left(n_{\mathrm{HI}} \approx 2 \times\right.$ $10^{19} \mathrm{~cm}^{-2}$ ). Comparing this spectrum with a sample of PSPC spectra of hot white dwarf stars (Jordan et al. 1994), we conclude that the X-ray spectrum of HR 2875 definitely bears the signature of a hot, noninteracting white dwarf with, most probably, a hydrogen-rich (DA) atmosphere.

On the other hand, ROSAT observations of OB stars show that the spectral type B1 defines a dividing line for early-type star X-ray emission (Berghöfer et al. 1997). It is accepted that $\mathrm{X}$-ray emission in early-type stars is produced by shocks in massive stellar winds. The ratio between bolometric and X-ray luminosity observed for HR $2875, \log \left(L_{\mathrm{X}} / L_{\text {bol }}\right)=-4.84$, exceeds the canonical value of $\log \left(L_{\mathrm{X}} / L_{\mathrm{bol}}\right) \sim-7$ for $\mathrm{O}$ stars. The X-ray emission from HR 2875 cannot be explained by shocks in the stellar wind; other X-ray-emitting mechanisms are not known for B stars.

\subsection{Ultraviolet and Optical}

EUV detection of the B star HR 2875 was reported in the ROSAT Wide Field Camera (WFC) all-sky survey and then in the EUVE all-sky survey; the ROSAT WFC observations occurred between 1990 October 13 and 23, while the EUVE observations occurred between 1992 October 23 and November 2. Table 1 summarizes the photometric EUV/soft X-ray measurements. EUVE spectroscopic observations of HR 2875 were obtained between 1996 April 5 and 14 for a total exposure of $113,000 \mathrm{~s}$. The star was detected only in the short-wavelength spectrometer $(\mathrm{SW})$. Because of the poor signal-to-noise ratio 


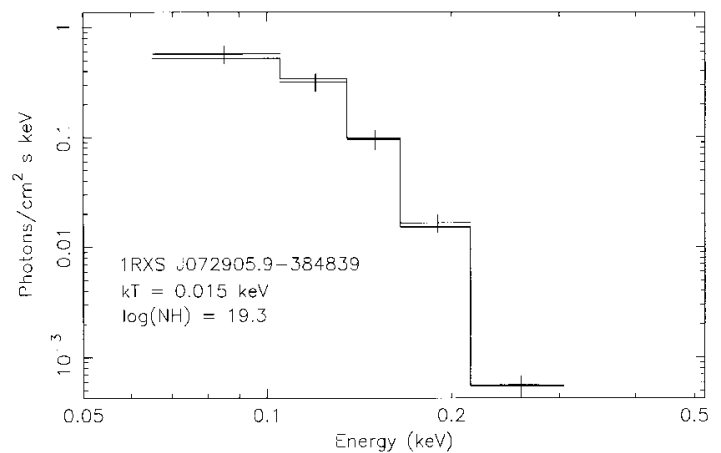

FIG. 1.-ROSAT PSPC energy distribution in the range 0.1-0.3 keV (41-124 $\AA$ ). The spectrum is characteristic of a hot, noninteracting, white dwarf observed toward a low column density in the interstellar medium.

achieved during this observation, the spectrum was reconstructed with 5 A bins. Finally, we obtained two high-dispersion IUE spectra of HR 2875 (LWP 29521 and SWP 52272) from the archives at Goddard Space Flight Center. We reconstructed the spectra with $2.5 \AA$ wavelength bins; Figure 2 presents the ultraviolet energy distribution between 70 and $3250 \AA$.

We obtained a series of high-dispersion spectra of HR 2875 using the Coudé Auxiliary Telescope (CAT) and Hamilton echelle spectrograph at Lick Observatory on 1997 January $8-11$, February 6 and 9, and March 12 . We used a $2048 \times$ 2048 CCD binned $2 \times 2$ with a dispersion of $0.054 \AA$ pixel $^{-1}$ at $\mathrm{H} \alpha$; the spectral resolution is $7.5 \mathrm{~km} \mathrm{~s}^{-1}$. The spectra were wavelength calibrated using Th-Ar comparison lamps. We observed HR 2875 at low air mass at the coudé focus of the 74 inch $(1.9 \mathrm{~m})$ telescope at Mount Stromlo Observatory (MSO) on 1997 April 24 and 25. We used the 32 inch $(81 \mathrm{~cm})$ camera and a 600 lines $\mathrm{mm}^{-1}$ grating resulting in a dispersion of 0.163 $\AA$ pixel $^{-1}$ at $\mathrm{H} \varepsilon$ in the third order and $0.243 \AA$ pixel $^{-1}$ at $\mathrm{H} \alpha$ in the second order. We established the wavelength scale using $\mathrm{Cu}-\mathrm{Ar}$ comparison lamps. We obtained $300 \mathrm{~s}$ exposures of the high Balmer line series ( $\mathrm{H} \delta-\mathrm{H} 9,3798-4132 \AA$ ) and $\mathrm{H} \alpha$ spectral range (6324-6820 $\AA$ ); we eliminated overlapping orders using BG12 and GG495 filters, respectively. The Lick and Stromlo images were bias subtracted and flat-fielded using a quartz lamp, and the spectra were extracted using standard NOAO's IRAF procedures.

\section{A B5 V PLUS DA WHITE DWARF BINARY}

\subsection{The B Star}

HR 2875 is classified as B5 V, corresponding to $T_{\text {eff }} \approx$ $15,000 \mathrm{~K}$, and $\log g \approx 4$. Figure 2 shows the ultraviolet (70-3200 A) spectrum observed with IUE and EUVE; the medium-resolution $E U V E$ spectrum confirms the presence of a white dwarf companion to the B star. Figure 3 shows high-

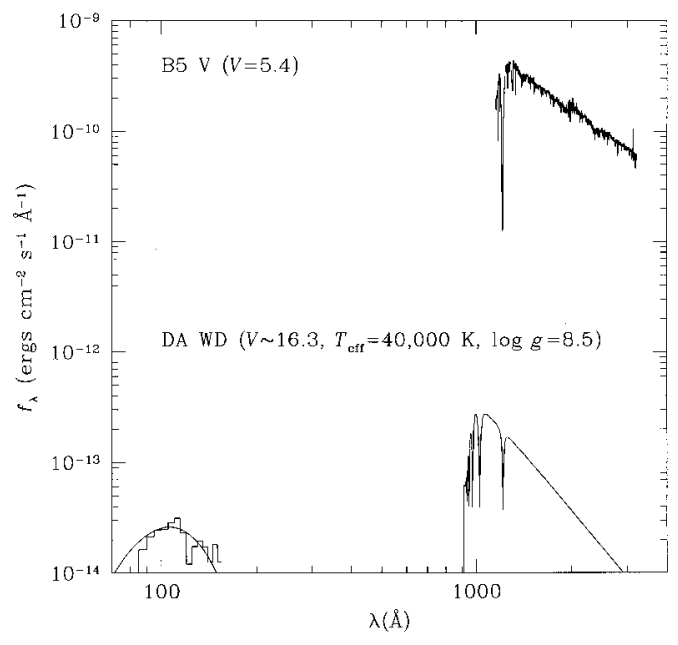

FIG. 2.-Ultraviolet spectrum of a composite B5 V star plus a hot white dwarf. EUV emission (80-150 A) demonstrates the presence of a hot white dwarf companion to the B star HR 2875 . The white dwarf is approximately 11 mag fainter in the optical range. The white dwarf EUV emission is compared with a representative model atmosphere $\left(T_{\text {eff }}=40,000 \mathrm{~K}, \log g=8.5\right)$ scaled to a distance of $177 \mathrm{pc}$ and theoretical white dwarf radius. The EUV spectral range is attenuated by a neutral hydrogen absorption in the local ISM $\left(n_{\mathrm{HI}}=3 \times 10^{19} \mathrm{~cm}^{-2}\right)$.

dispersion spectra of $\mathrm{H} \alpha$ and the $\mathrm{Si}$ II $\lambda 3858$ triplet obtained at Mount Stromlo. Hiltner et al. (1969) noted strong optical Si II lines that appeared peculiar: the MSO high-dispersion optical spectra show narrow lines of the Si II $\lambda 3858$ triplet and Si II $\lambda 4130$ doublet in the $\mathrm{H} \delta-\mathrm{H} 9$ spectral range. Surprisingly, the shape of the Si II lines varies with time and, synchronized with $\mathrm{H} \alpha$ emission, appeared to split during the April 24 observation. The lines appeared normal on April 25, and the equivalent width measurement of Si II $\lambda 4128.067, W=157 \mathrm{~m} \AA$, translates into an abundance between $\log (\mathrm{Si} / \mathrm{H})=-4.1\left(\xi=0 \mathrm{~km} \mathrm{~s}^{-1}\right)$ and $\log (\mathrm{Si} / \mathrm{H})=-4.8\left(\xi=6 \mathrm{~km} \mathrm{~s}^{-1}\right)$ using Becker \& Butler's (1990) non-LTE equivalent width calculations. Depending on the exact value of the microturbulence velocity, $\xi$, the Si abundance would be above or below the solar abundance $[\log (\mathrm{Si} / \mathrm{H})=-4.5]$, but it remains comparable to other silicon abundance measurements in B stars (see a compilation in Adelman, Robinson, \& Wahlgren 1993). We note that Be stars are not known to show Si II emission lines.

Table 2 presents our radial velocity measurements probing timescales of hours, days, and months. The average radial velocity is $\left\langle\nu_{\mathrm{rad}}\right\rangle=24.9 \mathrm{~km} \mathrm{~s}^{-1}$ with a standard deviation of only $1.4 \mathrm{~km} \mathrm{~s}^{-1}(n=18)$. The value is in remarkable agreement with Campbell \& Moore (1928) who measured $\left\langle v_{\mathrm{rad}}\right\rangle=+26.0$ $\mathrm{km} \mathrm{s}^{-1}, \sigma\left(v_{\mathrm{rad}}\right)=6.3 \mathrm{~km} \mathrm{~s}^{-1}$. Therefore, the system shows no measurable radial velocity variations. On the other hand, the B star clearly displays variable line profiles on a timescale of

TABLE 1

EUV and Soft X-Ray Measurements of HR 2875

\begin{tabular}{|c|c|c|c|c|c|}
\hline \multicolumn{2}{|c|}{$E U V E^{a}$} & \multicolumn{2}{|c|}{ ROSAT $\mathrm{WFC}^{\mathrm{b}}$} & \multicolumn{2}{|c|}{ ROSAT PSPC } \\
\hline Survey $100 \AA$ & DS $100 \AA$ & S1 & S2 & PSPC & $H R 1$ \\
\hline $0.094 \pm 0.012 \ldots$ & $0.199 \pm 0.016$ & $0.061 \pm 0.014$ & $0.108 \pm 0.016$ & $0.50 \pm 0.05$ & $-1.00 \pm 0.02$ \\
\hline
\end{tabular}

${ }^{\text {a }}$ EUVE all-sky survey observations in the Lexan bandpass (Survey $100 \AA$ ) and Guest Observer pointed observation using Deep Survey instrument and the Lexan bandpass (DS $100 \AA$ ).

${ }^{\mathrm{b}}$ ROSAT WFC all-sky survey observations at 90 (S1) and 160 (S2) $\AA$.

${ }^{\mathrm{c}}$ ROSAT PSPC hardness ratio defined as $H R I=(B-A) /(B+A)$, where $A$ is the count rate between 0.11 and $0.41 \mathrm{keV}$ and $B$ is the count rate between 0.52 and $2.01 \mathrm{keV}$. 


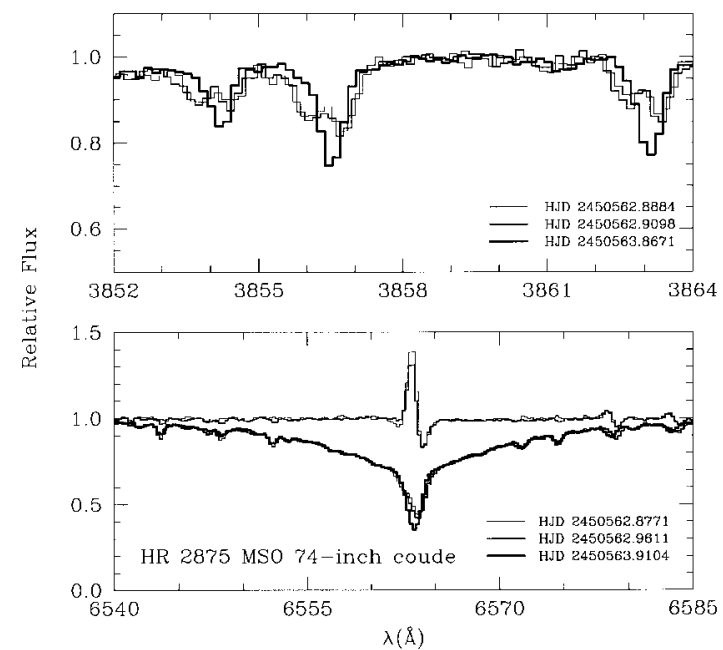

FIg. 3.-Coudé spectra of the B star HR 2875 showing emission reversals occurring on 1997 April 24 in the cores of $\mathrm{H} \alpha$, $\mathrm{He}$ I $\lambda 6678.15$ (bottom), and the Si II $\lambda 3858$ triplet lines (top). We divided the $\mathrm{H} \alpha$ spectra obtained on April 24 with the spectrum obtained on April 25 and plotted the resulting emission features.

1 day, which may be linked to the presence of a white dwarf companion. We note that Buscombe \& Morris (1958) report large radial velocity variations in four measurements (extrema: -5 and $+40 \mathrm{~km} \mathrm{~s}^{-1}$ ); their data may have been affected by variations in the line shape (Fig. 3), which, until now, were unnoticed. Future observations should be aimed at establishing a periodicity for these variations.

\subsection{The White Dwarf}

Because the B star dominates the optical and far-ultraviolet (FUV) spectral range, we must then determine the solid angle subtended by the white dwarf, assuming a distance and a radius for the compact object. Fortunately, the distance to the B star is known (164-196 pc), and the white dwarf radius is strictly a function of age and stellar mass, which are easily converted into effective temperature and surface gravity measurements adopting the evolutionary mass-radius relations of Wood (1995):

$$
R_{\mathrm{wD}}=f(\text { Age, } M)=f\left(T_{\text {eff }}, g\right) \text {. }
$$

Figure 4 shows an analysis of the ROSAT/EUVE data in the ( $\left.T_{\text {eff }}, \log g, \log n_{\mathrm{HI}}\right)$ parameter space, where $n_{\mathrm{H}}$ is the neutral hydrogen column density in the local interstellar medium (ISM), adopting pure hydrogen model atmospheres. Likelihood contours at $66 \%(1 \sigma), 90 \%$, and $99 \%$ are projected in the $\left(T_{\text {eff }}\right.$, $\log g)$ and $\left(T_{\text {eff }}, \log n_{\mathrm{HI}}\right)$ planes. Assuming a surface gravity in the range $7.0 \leq \log g \leq 9.5$, the photometric data alone constrain well the white dwarf parameters:

$$
\begin{aligned}
T_{\text {eff }} & =34.0-52.5 \times 10^{3} \mathrm{~K}, \\
n_{\mathrm{HI}} & =0.8-4.0 \times 10^{19} \mathrm{~cm}^{-2} .
\end{aligned}
$$

\begin{tabular}{|c|c|c|}
\hline HJD $2,450,000+$ & $\begin{array}{c}v \\
\left(\mathrm{~km} \mathrm{~s}^{-1}\right)\end{array}$ & Lines Measured \\
\hline 456.8639 & $\ldots$ & Emission cores \\
\hline 456.8764 & ... & Emission cores \\
\hline 456.8884 & $\ldots$ & Emission cores \\
\hline 457.8663 & +26.3 & $\mathrm{H} \alpha$ \\
\hline 457.8783 & +27.2 & $\mathrm{H} \alpha$ \\
\hline 457.9283 & +26.6 & $\mathrm{H} \alpha$ \\
\hline 458.9146 & +24.1 & $\mathrm{H} \alpha$ \\
\hline 458.9284 & +24.1 & $\mathrm{H} \alpha$ \\
\hline 459.8189 & +23.6 & $\mathrm{H} \alpha$ \\
\hline $459.8871 \ldots \ldots \ldots$ & +23.9 & $\mathrm{H} \alpha$ \\
\hline 459.9531. & +25.1 & $\mathrm{H} \alpha$ \\
\hline 485.7201 . & $\ldots$ & Emission cores \\
\hline 485.7530 & $\ldots$ & Emission cores \\
\hline 485.7785 . & $\ldots$ & Emission cores \\
\hline 485.8027 & $\ldots$ & Emission cores \\
\hline 485.8242 & $\ldots$ & Emission cores \\
\hline 488.7189 & +25.1 & $\mathrm{H} \alpha$ \\
\hline 488.7415 & +25.0 & $\mathrm{H} \alpha$ \\
\hline $488.7642 \ldots \ldots \ldots$ & +25.0 & $\mathrm{H} \alpha$ \\
\hline 488.7867 & +26.3 & $\mathrm{H} \alpha$ \\
\hline 488.8100 & +26.7 & $\mathrm{H} \alpha$ \\
\hline 519.6968 & +22.5 & $\mathrm{H} \alpha$ \\
\hline $519.7140 \ldots$ & +22.9 & $\mathrm{H} \alpha$ \\
\hline $519.7365 \ldots \ldots \ldots$ & +22.9 & $\mathrm{H} \alpha$ \\
\hline $562.8771 \ldots \ldots \ldots$ & $\ldots$ & Emission cores \\
\hline $562.8884 \ldots$ & .. & Emission cores \\
\hline 562.9098 . & $\ldots$ & Emission cores \\
\hline $562.9611 \ldots \ldots \ldots$ & $\ldots$ & Emission cores \\
\hline $563.8671 \ldots$ & +26.0 & $\mathrm{H} \gamma-\mathrm{H} 8, \mathrm{Si}$ II \\
\hline 563.9104 . & +25.0 & $\mathrm{H} \alpha$ \\
\hline
\end{tabular}

Restricting the surface gravity to a range corresponding to a
TABLE 2

massive white dwarf, i.e., $9.0 \leq \log g \leq 9.5$, we then find that

$$
\begin{aligned}
& T_{\text {eff }}=42.0-52.5 \times 10^{3} \mathrm{~K}, \\
& n_{\mathrm{HI}}=0.8-3.0 \times 10^{19} \mathrm{~cm}^{-2} .
\end{aligned}
$$
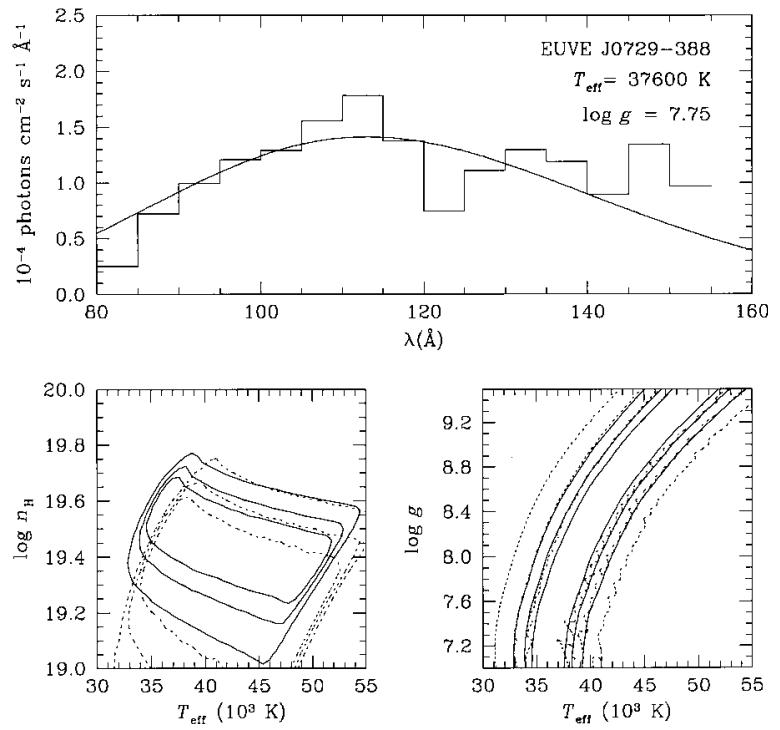

Fig. 4.-Model atmosphere analysis of the EUVE photometric and spectroscopic data and ROSAT WFC photometric data in the $\left(T_{\text {eff }}, g, n_{\mathrm{H}}\right)$ parameter space (bottom). Solutions to the photometric (dashed lines) and spectroscopic (solid lines) analyses are shown with 66\%,90\%, and 99\% confidence contours in the $\left(T_{\text {eff }}, \log g\right.$ ) plane (bottom right) and in the $\left(T_{\text {eff }}, \log n_{\mathrm{H}}\right)$ plane (bottom left). The models are assumed pure in hydrogen and are normalized to a distance of $177 \mathrm{pc}$ and theoretical white dwarf radii (Wood 1995). The best model atmosphere fit is presented with the EUVE spectrum (top). 
The assumption of a pure hydrogen composition for the atmosphere of the white dwarf is debatable, considering the relatively high effective temperature range deduced for the star. Recent ROSAT soft X-ray and EUV measurements (see Wolff, Jordan, \& Koester 1996; Barstow et al. 1993) confirm the prevalence of trace heavy elements among DA white dwarfs with $T_{\text {eff }} \geq 40,000 \mathrm{~K}$. The low signal-to-noise ratio achieved during the EUV spectroscopic observation does not allow us to ascertain the presence of heavy elements in the atmosphere of the white dwarf (Fig. 3).

Figure 4 also shows an analysis of the EUVE spectroscopic data. The range of solutions obtained is consistent with the analysis of photometric data:

$$
\begin{aligned}
& T_{\text {eff }}=34.5-52.0 \times 10^{3} \mathrm{~K}, \\
& n_{\mathrm{HI}}=1.8-5.0 \times 10^{19} \mathrm{~cm}^{-2},
\end{aligned}
$$

again assuming that $7.0 \leq \log g \leq 9.5$. Fixing the surface gravity at $\log g=8.0$ and $\log g=9.0$, the effective temperature is in the ranges

$$
\begin{aligned}
& T_{\text {eff }}=37.0-40.0 \times 10^{3} \mathrm{~K}\left(M_{\mathrm{wD}} \approx 0.7 M_{\odot}\right), \\
& T_{\text {eff }}=42.5-46.5 \times 10^{3} \mathrm{~K}\left(M_{\mathrm{WD}} \approx 1.2 M_{\odot}\right),
\end{aligned}
$$

respectively. Following the same procedure, the ROSAT PSPC spectrum corresponds to almost identical effective temperature ranges:

$$
\begin{aligned}
& T_{\text {eff }}=37.5-43.0 \times 10^{3} \mathrm{~K}\left(M_{\mathrm{wD}} \approx 0.7 M_{\odot}\right), \\
& T_{\text {eff }}=41.8-47.0 \times 10^{3} \mathrm{~K}\left(M_{\mathrm{wD}} \approx 1.2 M_{\odot}\right) .
\end{aligned}
$$

The $\mathrm{H}$ I column density in the local ISM is limited to $n_{\mathrm{HI}} \leq$ $5.6 \times 10^{19} \mathrm{~cm}^{-2} \quad\left(\log g=8\right.$ models) and $n_{\mathrm{HI}} \leq 3.4 \times$ $10^{19} \mathrm{~cm}^{-2}$ ( $\log g=9$ models), in agreement with the EUV data. These ranges of effective temperatures, for either masses, are generally characterized by a low heavy element abundance $(Z)$ in white dwarf atmospheres. However, should the white dwarf possess a mass in excess of $1.2 M_{\odot}$ and resemble the massive DA white dwarf GD $50\left(T_{\text {eff }}=43,000 \mathrm{~K}, \log g=\right.$ 9.2; Vennes et al. 1997), it may then share also its large helium abundance $\left(Y=2.4 \times 10^{-4}\right.$; Vennes, Bowyer, \& Dupuis 1996). So far, the spectroscopic properties of GD 50 appear unique among hot white dwarf stars, but they may also be a common characteristic of massive white dwarfs.

\section{SUMMARY}

In summary, we find that a hot DA white dwarf with atmospheric parameters representative of the EUV-selected population of white dwarf stars (Vennes et al. 1997) provides an excellent interpretation of the EUV and soft X-ray observations of the B star HR 2875. The atmospheric parameters $\left(T_{\text {eff }}, g, Y\right.$, $Z$ ) remain uncertain; additional EUV soft X-ray observations are needed to characterize the white dwarf fully. The B star itself is peculiar, showing variable $\mathrm{H} \mathrm{I}, \mathrm{He} \mathrm{I}$, and $\mathrm{Si}$ II line profiles with unknown periodicity, and it is now found to hide a white dwarf companion. The proximity of the B star and the white dwarf $(\leq 5$ ".5), source of the EUV/soft X-ray emission, rules out a chance alignment and imposes a physical association.

The association of a white dwarf with a B5 V star extends the mass of main-sequence stars known to evolve through the asymptotic giant branch to at least $6 M_{\odot}$. It is usually believed such objects could be as massive as $8 M_{\odot}$. Nomoto (1984) extended this range to $8-11 M_{\odot}$ main-sequence stars, possible progenitors of massive $\mathrm{O}-\mathrm{Ne}-\mathrm{Mg}$ white dwarfs. It is therefore critical to determine the surface gravity, and hence the mass, of the white dwarf in HR 2875. Such a measurement will be possible with the spectrometers on board the Advanced X-Ray Astrophysics Facility (AXAF) using the extreme sensitivity of the soft X-ray continuum of hot DA white dwarfs to surface gravity (Vennes 1992).

The peculiarity of the B star will be investigated further at MSO, with the particular aim of determining strict periodicity for the line variations. Emission features in the B-star spectrum raise the possibility of interaction, irradiation or mass exchange, between the binary components.

This work is supported by NASA grant NAG5-2636 and NASA contract NAS5-30180. T. W. B. acknowledges support from the Alexander von Humboldt Stiftung $(\mathrm{AvH})$ in the form of a Feodor Lynen Fellowship.

\section{REFERENCES}

Adelman, S. J., Robinson, R. D., \& Wahlgren, G. M. 1993, PASP, 105, 327 Barstow, M. A., et al. 1993, MNRAS, 264, 16

Becker, S. R., \& Butler, K. 1990, A\&AS, 84, 95

Berghöfer, T. W., Schmitt, J. H. M. M., \& Cassinelli, J. P. 1996, A\&AS, 118, 481

Berghöfer, T. W., Schmitt, J. H. M. M., Danner, R., \& Cassinelli, J. P. 1997, A\&A, 322, 167

Blöcker, T. 1995, A\&A, 297, 727

Bowyer, S., Lampton, M., Lewis, J., Wu, X., Jelinsky, P., \& Malina, R. F. 1996, ApJS, 102, 129

Buscombe, W., \& Morris, P. M. 1958, MNRAS, 118, 609

Campbell, W. W., \& Moore, J. H. 1928, Pub. Lick Obs., 16, 113

Hiltner, W. A., Garrison, R. F., \& Schild, R. E. 1969, ApJ, 157, 313

Jordan, S., Wolff, B., Koester, D., \& Napiwotzki, R. 1994, A\&A, 290, 834
Motch, C., Haberl, F., Dennerl, K., Pakull, M., \& Janot-Pacheco, E. 1997, A\&A, 323, 853

Nomoto, K. 1984, ApJ, 277, 791

Pye, J. P., et al. 1995, MNRAS, 274, 1165

Vassiliadis, E., \& Wood, P. R. 1993, ApJ, 413, 641

Vennes, S. 1992, ApJ, 390, 590

Vennes, S., Bowyer, S., \& Dupuis, J. 1996, ApJ, 461, L103

Vennes, S., Thejll, P., Génova-Galvan, R., \& Dupuis, J. 1997, ApJ, 480, 714

Voges, W., et al. 1997, A\&A, in press

Wagenhuber, J., \& Weiss, A. 1994, A\&A, 290, 807

Wolff, B., Jordan, S., \& Koester, D. 1996, A\&A, 307, 149

Wood, M. A. 1995, in White Dwarfs, ed. D. Koester \& K. Werner (Berlin: Springer), 41 